\title{
SMM CORONAGRAPH OBSERVATIONS OF PARTICULATE CONTAMINATION
}

\author{
O.C. ST.CYR, and
}

T. WARNER

High Altitude Observatory, National Center for Atmospheric Research ${ }^{1}$

P.O. Box 3000 , Boulder, CO 80307

\begin{abstract}
Some recent images taken by the white light coronagraph telescope aboard the Solar Maximum Mission (SMM) observatory show bright streaks that are apparently caused by particles associated with the spacecraft. In this report we describe these observations, and we analyze the times of their occurrence. We demonstrate that the sightings occur most often near SMM's orbital dawn, and we speculate that thermal shock is the mechanism that produces the particles. Although these sightings have not seriously affected the coronagraph's scientific operations, the unexpected passage of bright material through the field of view of sensitive spaceborne telescopes can lead to data loss or, in some cases, serious detector damage. The topic of space debris has become a significant concern for designers of both manned and unmanned orbiting platforms. The returned samples from the SMM spacecraft and the observations reported here provide a baseline of experience for future orbital platforms that plan long duration missions.
\end{abstract}

\section{INTRODUCTION}

The Solar Maximum Mission (SMM) observatory was launched February 14, 1980, from Cape Canaveral into a $574 \mathrm{~km}$ altitude circular orbit inclined 28.5 degrees to the equator. Among SMM's primary science objectives have been the study of the dynamics of solar flares and the study of solar magnetic fields associated with the flare phenomenon. SMM's payload consisted of eight instruments that provided broad spectral coverage of radiation produced by solar flares, to include gamma radiation, hard and soft x-rays, ultraviolet, and the sun's white light corona. Five of these instruments produced images and required a stable platform for accurate (several arc second) pointing; the other three instruments can be considered unpointed. A detailed description of the SMM instruments and the mission objectives was reported in Solar Physics $(\underline{65}, 1980)$.

1. The National Center for Atmospheric Research is supported by the National Science Foundation 
SMM was the first multi-mission modular spacecraft, and it was designed to be retrieved by the space shuttle. Its major subsystems were engineered for inorbit replacement with relative ease if that need ever arose. In fact, nine months after launch, the SMM attitude control system malfunctioned, and stable pointing was no longer possible. Also, in the weeks prior to the attitude control system malfunction, the white light coronagraph/polarimeter (C/P) suffered an electronics failure that rendered the instrument inoperative.

The Challenger space shuttle was launched on April 6,1984, to attempt a repair of SMM. The mission was successful, and both the spacecraft attitude control system and the C/P Main Electronics Box were replaced. Details of the retrieval, repair, and redeployment of SMM by the shuttle crew have been documented recently by Woodgate and Maran (1987).

From repair until December, 1986, the C/P telescope obtained $\sim 120,000$ images of the solar corona (Figure 1a). A fraction of a percent $(173 / 120,000$ or $0.14 \%$ ) of the $\mathrm{C} / \mathrm{P}$ images have been marred by the presence of particles that appear to be in the near field of the instrument (Figure 1b). These appear as bright streaks, and occasionally (in 52/173 or 30\% of the contaminated images) several streaks appear in a single image. In a few instances $(21 / 173$ or $12 \%)$, consecutive images taken three minutes apart have shown evidence of the contamination.

We will show in this report that the particles are apparently associated with the SMM spacecraft. The presence of the particles has on at least one occasion (May 31, 1986) "confused" the SMM attitude control system when the sun sensors registered saturation. The spacecraft computer attempted to track these bright objects, and the spacecraft entered "safehold" mode. In this mode of operation the control of the spacecraft's attitude is taken away from the onboard computer. Unexpected entry into this mode results in a significant loss of science data since only minimal attitude control is available. Moreover, the period of less accurate pointing represents a danger for sensitive detectors such as that used by the coronagraph. Reconfiguration of the spacecraft and instruments for normal science operations also requires significant time and communication with the spacecraft.

We initiated this study after the May 31,1986 , incident when we realized the potential danger to the spacecraft and to the payload. In particular, we were concerned that the presence of debris near the aperture of the coronagraph could cause scattering of full sunlight into the telescope tube, and such a circumstance could render the instrument useless for imaging the faint solar corona. An initial presentation of these sightings was made by St.Cyr et al (1987). In this report we first describe the coronagraph telescope and the relevant observations obtained between June, 1984, and December, 1986. We also present an elementary analysis of the occurrence of the sightings, and we conclude with a discussion.

\section{THE CORONAGRAPH/POLARIMETER (C/P)}

The coronagraph is an instrument that produces an artificial eclipse of the sun's disk, thereby revealing the faint outer atmosphere of the sun--the corona. The C/P telescope aboard SMM uses a complex arrangement of internal and external occulting methods to block out the sun's disk and image the sun's outer corona. Details concerning this instrument have been documented in MacQueen 
et al (1980). The instrument's unobstructed field of view extends from 1.7 solar radii to 5 solar radii (the field of view is about a degree and a quarter on a side). The instrument produces images of one quadrant of the corona in each exposure--on the accompanying figures an arrow indicating North and a dotted circle representing the size and location of the solar disk have been added to the images. Numerous electronic artifacts appear in each image, and these are described in the caption to Figure $1 \mathrm{a}$. While these stable artifacts can be removed by image processing techniques, we will present only the unprocessed data with this report.

The detector for $\mathrm{C} / \mathrm{P}$ is an SEC vidicon tube. The brightness of the corona is typically $10 \mathrm{E}(-9)$ that of the solar disk; scattering due to stray light in the coronagraph is less than $3 \times 10 \mathrm{E}(-10)$ of the radiance of the solar disk in the outer field of view. All of the images contaminated with particle streaks were obtained through a broadband filter (half-power bandpass 5000 to 5350 Angstroms). The exposure time for each image was typically 6 or 8 seconds. The images were digitized and stored on one of three high density tape recorders aboard the spacecraft. The tape recorders were dumped to a NASA ground tracking station or through the Tracking and Data Relay Satellite System several times each 24 hour day.

During SMM's first period of operation (February, 1980 until the instrument experienced the electronics failure in September, 1980) $\sim 30,000$ images were obtained by the coronagraph. The bright streaks that we report here as contamination were seen on only two occasions during the 1980 operations. The instrument was not operated again until after the repair of SMM in 1984. $\mathrm{C} / \mathrm{P}$ operations resumed in early June, 1984 . Following the 1984 repair, images were typically taken at orbital sunrise and sunset; most of the desired solar science can be obtained by observing only at orbital sunrise and sunset. Scientific interpretation of these recent observations has been presented by Hundhausen (1987).

\section{ANALYSIS OF PARTICLE SIGHTINGS}

Out of the $\sim 120,000$ images acquired by the coronagraph between June 8 , 1984 , and December 7, 1986, only $173(0.14 \%$ of the total) show evidence of bright streaks. All of the contaminated images were discovered by visual inspection of the individual frames on a video display during the normal data reduction process of cataloging and commenting. We note that the images reported in this study represent only the brightest sightings, and thus the numbers used here are certainly a lower limit to the total number of contaminated images that may have been detected by this instrument. The time-consuming process of digitally "differencing" images (ie., a pixel-by-pixel subtraction from an earlier image) is not done routinely, but we suspect that more contaminated images would be found by this process. Therefore the sightings reported here have brightness well above some undetermined instrumental threshold for detection of space debris. As one measure of the sensitivity of the instrument we note that stars of astronomical magnitude +5 can typically be identified in the field of view of the undifferenced data. 
We have analyzed the occurrence of the particle sightings as a function of several parameters. In Figure 2, we show the number of sightings as a function of elapsed time. Figure $2 \mathrm{a}$ shows the normalized probability for occurrence, and Figure $2 \mathrm{~b}$ displays the absolute number of sightings versus elapsed time since repair. We have determined the occurrence probability for the debris sightings as the simple ratio of contaminated images to total number of images for each 30 day interval. A line marking the average for each set is also displayed.

From Figure 2, it appears that the probability of the sightings may have increased in early 1986 . We are aware of two possible events or series of events immediately prior to January, 1986 . In late December, 1985 , operations personnel accelerated the testing schedule for the SMM observations of Comet Halley. The testing (March, 1985 to January, 1986) and the comet observations (January-February, 1986) required repeated slews of the spacecraft away from the Sun (up to 40 degrees) for durations of up to one hour. It is important to note that during nominal solar observations SMM is always pointed toward the Sun--even during orbital night--and that surfaces of the SMM bus and payload that had not previously been illuminated were subjected to sunlight during these exercises. However, slews of SMM off the Sun line cannot account for all of the particle sightings, since the sightings began shortly after operations commenced in 1984, and the sightings continued through 1986 after the offpointing exercises were terminated. Also, the specific times of the sightings do not correlate with times of the offpointing exercises during 1985.

A second event that could be related to the early 1986 increase in debris sightings was the destruction of the P78-1 satellite by the U.S. Air Force in September, 1985. Prior to that anti-satellite test, the P78-1 satellite was in a polar orbit, and its perigee was a few 10's of kilometers above SMM's apogee. Again, this event cannot account for all of the SMM particle sightings, since the sightings began in mid-1984.

We have also investigated the debris sightings as a function of SMM's orbital phase. In Figure 3a, we have plotted the normalized probability of sightings in each 10 minute interval with respect to the time since orbital sunrise. In Figure $3 \mathrm{~b}$, we show the absolute numbers of these same data. In producing this graph we have combined the data for the two 10 minute bins near orbital noon (21-40 minutes after orbital sunrise), since few images were taken during this 20 minute interval. As mentioned earlier, following the repair of SMM the instrument was most often operated in a synoptic "patrol" mode. In this mode of operation 4 images of the corona (the four quadrants) were acquired twice each orbit-in the first 15 minutes following orbital dawn and in the last 15 minutes prior to orbital sunset.

From Figure 3, it is apparent that both the absolute number of particle sightings and their occurrence probability are maximum near orbital sunrise and fall to a minimum near orbital sunset. Debris from other satellites at higher altitudes produces a continual flux of particles as fragments drift to lower altitudes because of atmospheric drag (e.g., Kessler, 1985). However, the strong dependence of the contaminated images on SMM's orbital phase suggests that these particles are associated with the spacecraft itself.

Since the sightings occur preferentially at orbital dawn we must consider what is different at that location in the orbit. The most obvious sunrise associated event is the thermal shock to the spacecraft as it leaves the darkness of the Earth's shadow and enters full sunlight over a matter of several tens of seconds. 
Shown in Figure 4 is the number of sightings versus the day of year, summed for 10 day intervals in 1984/85/86. Again, both the normalized occurrence probability and the absolute number of sightings are shown. We have analyzed the sightings as a function of day-of-year to investigate the possibility that recurrent meteor showers might be related to the particle sightings. However, the sightings appear to be random when plotted against calendar date. Further, the times of 19 principal meteor showers (as listed in Norton's Star Atlas) do not correspond to the peaks in this day-of-year graph.

We have been able to determine the sub-spacecraft latitude and longitude for 135 of the contaminated images, and these are shown in Figure 5. We have not determined the sub-spacecraft coordinates for each of the 120,000 images taken since 1984, so only the absolute numbers of contaminated images are shown. From Figure 5, we note that the sightings do not appear to correlate with SMM's passage through the South Atlantic Anomaly (SAA).

In Figure 6, we have summed the number of contamination sightings along 2 degree intervals of sub-spacecraft latitude and 10 degree intervals of subspacecraft longitude. While there does not appear to be a dependence on subspacecraft longitude, the sightings do show a preference for high sub-spacecraft latitudes (both positive and negative).

The apparent dependence of the number of sightings on sub-spacecraft latitude requires elaboration. We suggest that this effect, seen in Figure 6a, may actually relate to the direction of the spacecraft's instantaneous velocity. The magnitude of the sunward component of SMM's velocity vector is maximum at orbital dawn when the sub-spacecraft latitude is near the value of the orbital inclination (private communication, D. Kulp, SMM Flight Dynamics Facility, 1987). Although the Earth's atmosphere is a tenuous medium at the altitude of SMM, the fluence of the atmosphere against the SMM instrument faceplate should be maximum when orbital dawn occurs and the sub-spacecraft latitude is \pm 28 degrees. Also, following orbital dawn the density of the atmosphere increases as it is heated by the sun. These effects, combined with the thermal shock to the spacecraft at orbital sunrise, may explain the dependence found for dawn and sub-spacecraft latitude.

We have investigated further the concept of the atmospheric fluence on the spacecraft faceplate. The density of the upper atmosphere at the altitude of SMM (or any spacecraft in low Earth orbit) depends upon solar activity. Therefore we have also considered solar activity conditions during this time interval. We have used daily measurements of the $2800 \mathrm{MHz}(10.7 \mathrm{~cm})$ solar full disk flux as an indicator of the influence of solar activity on atmospheric density. However, we found no apparent correlation between the number of particle sightings and the magnitude of the $2800 \mathrm{MHz}$ solar flux.

We have recognized at least two subsets of the 173 sightings. A small number of the $C / P$ sightings probably represent the passage of other artificial earth satellites (AES) through the instrument's field of view during an exposure. The AES should appear in the coronagraph images as single, narrow bright streaks, and such images can be found (15/173 or 9\%). However, it is not clear how one can distinguish between a bright particle that is only a few meters distant and an AES that is several thousand kilometers distant. Such an image is shown in Figure 7. The problem of unexpected AES passage through the field of view of a sensitive detector has been discussed for the Hubble Space Telescope by Shara and Johnston (1986). They concluded that there is some possibility that at 
least one of the instruments on Space Telescope could experience degradation or damage by the sighting of a bright AES.

Another subset of the sightings is composed of images having many (more than 10) bright streaks in a single picture. This subset contains 22 members $(22 / 173$ or $13 \%)$, and as an example we show the sighting associated with the May 31,1986 , spacecraft "safehold" in Figure 8. This subset has been analyzed as a function of the parameters discussed in the previous section. We have not included graphic analysis of this subset in this report, since it appears that these "worst cases" also follow the same patterns found for the 173 sightings.

\section{DISCUSSION}

If the particles sighted by the coronagraph are associated with the SMM spacecraft, we must address the problem of their source and their irradiance. Similar contaminated images have been detected by another spaceborne coronagraph. A much larger fraction (approximately 10\%) of the 35,000 photographic frames taken by the Skylab ATM coronagraph contain evidence of particulates in the environs of the spacecraft. In a study of the statistical frequency of the sightings, McGuire (1976) concluded that the sightings were most likely spheres of ice. Those particles were associated with expendables from Skylab, and they could be correlated to times when the astronauts were "active" (as opposed to sleeping) or when "water dumps" occurred. From densitometer scans of the bright streaks in the ATM coronagraph frames, Schuerman et al (1977) were able to determine the velocity components (less than 10 meters/sec) and the distribution of radii ( 5 to 100 microns) for particles that were within 200 meters of the telescope. They concluded that direct (forward) scattering of sunlight by particles larger than a few microns in radius could account for the irradiance seen in the contaminated Skylab coronagraph images.

The only "expendable" aboard SMM is a propane gas system for the X-Ray Polychromator (XRP) instrument. The rate of consumption of this gas by XRP is usually constant; however, there are brief intervals when the detectors must be refilled with the gas. We have investigated times of unusual activity for the XRP instrument during the 1984-1986 interval, but no coincidence with $\mathrm{C} / \mathrm{P}$ debris sightings was found. The excess propane from XRP is vented out of a pipe on the side of the spacecraft. An aluminum baffle was placed over this pipe during the SMM repair mission so that plasma would not flow into the instrument from outside the spacecraft. Although we have not rigorously studied the idea, it seems conceivable that the vented propane may have frozen on this baffle, and that at certain times the ice may sublimate as changes occur in the spacecraft environs.

Another possibility is that the particles could be the result of the presence of Challenger in SMM's orbit for several days (e.g., Bareiss et al, 1986); however, we might expect the frequency of particle sightings to decrease with time as the shuttle-related contamination decayed to lower altitudes. Immediately following the repair of SMM and C/P, extensive spacecraft and instrument check-outs were performed. Regular observations by $\mathrm{C} / \mathrm{P}$ did not resume until June 1 , approximately 45 days after the repair.

We consider paint to be the most likely possibility for the source of the particles. Recently Laurance and Brownlee (1986) have determined the 
meteoroid and debris flux from the impact craters in the returned SMM attitude control system thermal louvers. Similar analyses of the SMM thermal blanket material (Rietmeijer et al, 1986; Schramm et al, 1985; Kessler et al, 1985) have also concluded that the debris flux exceeded the natural meteoroid flux, and that paint constituents were a major contributor in the returned SMM samples. In fact, for small particles ( $\sim 10 \mathrm{E}-12 \mathrm{gm})$ the debris flux exceeded that of meteoroids by two orders of magnitude.

White paint is often used for thermal protection on spacecraft, and SMM has several painted surfaces. The instrument faceplate, which is always pointed toward the Sun, was painted with a special pre-oxidized paint known as MS-74. Other white paints (not pre-oxidized) were used for the aperture door of the coronagraph, the backside of the solar arrays, and the outer surface of the new attitude control module installed in 1984 during the SMM repair. Visual inspection by the astronauts who repaired the spacecraft revealed no significant deterioration of painted surfaces (G. Nelson, private communication, 1987), but it seems unlikely that this inspection would have detected particles as small as reported by Laurance and Brownlee (1986).

\section{CONCLUSIONS}

We report that the SMM white light coronagraph has detected bright particles in its field of view on 173 occasions (representing $0.14 \%$ of the images between June, 1984 and December, 1986). We believe that the particles are associated with SMM, as indicated by analysis of the spacecraft's orbital phase at the times of the sightings. We suggest that thermal shock to the spacecraft at orbital sunrise may be the physical mechanism causing the orbital phase dependence. In view of the results of analyses of returned parts from SMM, it seems likely that the sightings may be chips of paint or paint residue.

It appears that the sightings became more frequent in early 1986, although we cannot give a definitive reason for this. It may be that the increased cadence of off-sun slews by SMM for the observations of Comet Halley contributed to the abrupt rise in particle sightings. We also note that the destruction of the P78-1 spacecraft by the U.S. Air Force had occurred several months before the increase in sightings.

There appears to be a sub-spacecraft latitude dependence for the particle sightings. The sightings are apparently more frequent at the highest (absolute) values of sub-spacecraft latitude. We have noted that the maxima for the sunward component of the SMM velocity vector occurs when the high subspacecraft latitudes occur at orbital dawn. We speculate that two factors (thermal shock at orbital dawn and maximum flow of the tenuous upper atmosphere against the instrument faceplate) may be major contributors acting on the source for the particles.

\section{ACKNOWLEDGMENTS}

We appreciate the assistance of a number of individuals at $\mathrm{HAO}$, to include: R.H. Lee, S. Beck, A. Stanger, J. Burkepile, D. Kobe, S. Rosenberg, and K. Walsh. At Goddard Space Flight Center, W. Ousley, K. Rosette, and D. Douds 
of the Satellite Servicing Project have provided useful information and discussion. We are grateful to K. Henize and D. Kessler of Johnson Space Center for comments concerning the manuscript. The SMM coronagraph operations and data analysis are performed by HAO for NASA under contract S-04167D.

\section{REFERENCES}

Bareiss, L.E., Payton R.M., and Papazian, H.A. (1986). "Shuttle/Spacelab contamination environment and effects handbook", NASA Contractor Report 3993.

Hundhausen, A.J. (1987). "The origin and propagation of coronal mass ejections", Proceedings of the Sixth International Solar Wind Conference, NCAR/TN$306+$ Proc.

Kessler, D.J. (1985). "Orbital debris issues", Advances in Space Research, $\underline{5}$, Number 2, 3.

Kessler, D.J., Zook, H.A., Potter, A.E., McKay, D.S., Clanton, U.S., Warren, J.L., Watts, L.A., Schultz, R.A., Schramm, L.S., Wentworth, S.J., and Robinson, G.A. (1985). "Examination of returned Solar Max surfaces for impacting orbital debris and meteoroids". Proc. Lunar and Planetary Science Conf.,16, 434.

Laurance, M.R., and Brownlee, D.E. (1986) "The flux of meteoroids and orbital space debris striking satellites in low Earth orbit", Nature, $\underline{323}, 136$.

MacQueen, R.M., Csoeke-Poeckh, A., Hildner, E., House, L.L., Reynolds, R., Stanger, A., TePoel, H., and Wagner W.J. (1980). "The High Altitude Observatory coronagraph/polarimeter on the Solar Maximum Mission", Solar Physics, 65, 91.

McGuire, J.P. (1976). "Contamination from Skylab as determined from the solar coronagraph data", NASA TM X-73353.

Rietmeijer, F.J.M., Schramm, L.S., Barrett, R.A., McKay, D.S., and Zook, H.A. (1986). "An inadvertent capture cell for orbital debris and micrometeorites; the main electronics box thermal blanket of Solar Maximum satellite", Adv. Space Res. 6, No.7, 145.

Shara, M.M., and Johnston, M.D. (1986). "Artificial earth satellite crossing the fields of view of, and colliding with orbiting space telescopes", PASP, $\underline{98}$, 814.

Schramm, L.S., McKay, D.S., Zook, H.A., and Robinson, G.A. (1985). "Analysis of micrometeorite material captured by the Solar Max", Proc. Lunar and Planetary Science Conf., $16,736$.

Schuerman, D.W., Beeson, D.E., and Giovane, F. (1977). "Coronagraphic technique to infer the nature of the Skylab particulate environment". Applied Optics, 16, 1591.

St.Cyr, O.C., Warner, T., Beck, S., and Burkepile, J. (1987). "SMM coronagraph observations of contamination in low Earth orbit", Eos, 68, 378.

Woodgate, B.E., and Maran, S.P. (1986). "The Solar Maximum Mission repair--lessons learned", Space Station Automation II, SPIE Conference Proc.,729, 202. 


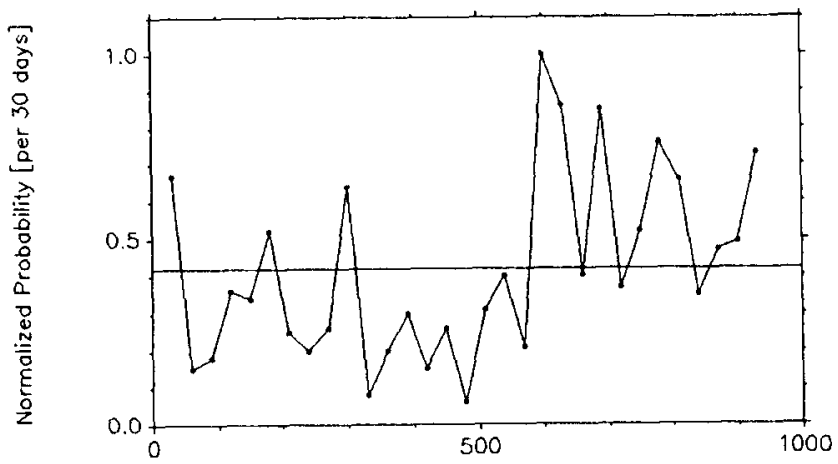

(a)

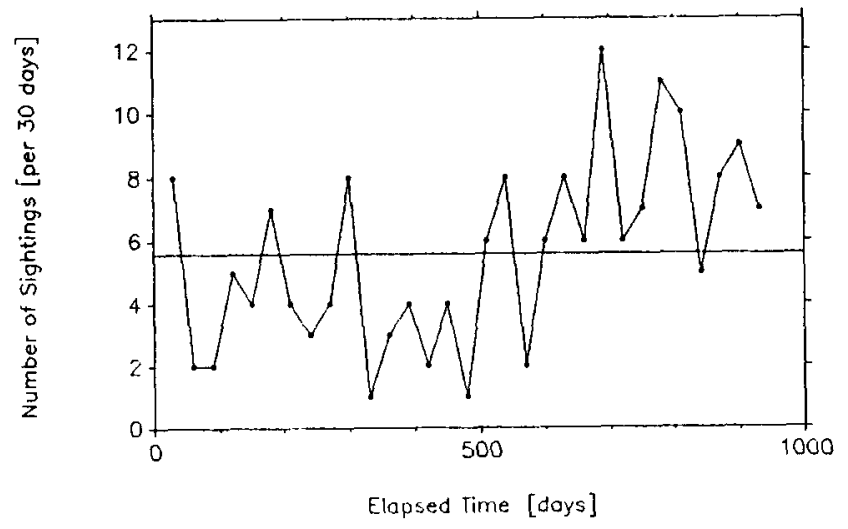

(b)

FIGURE 1 - Sightings versus Mission Elapsed Time

(a) Thirty day averages of normalized occurrence probability for the sightings have been plotted against elapsed time since operations resumed in June, 1984. The average normalized probability per interval (0.42) is also shown.

(b) As in 2(a) but absolute number of particle sightings summed over thirty day intervals. The average number of sightings (5.6 sightings per 30 day interval) is also marked. 


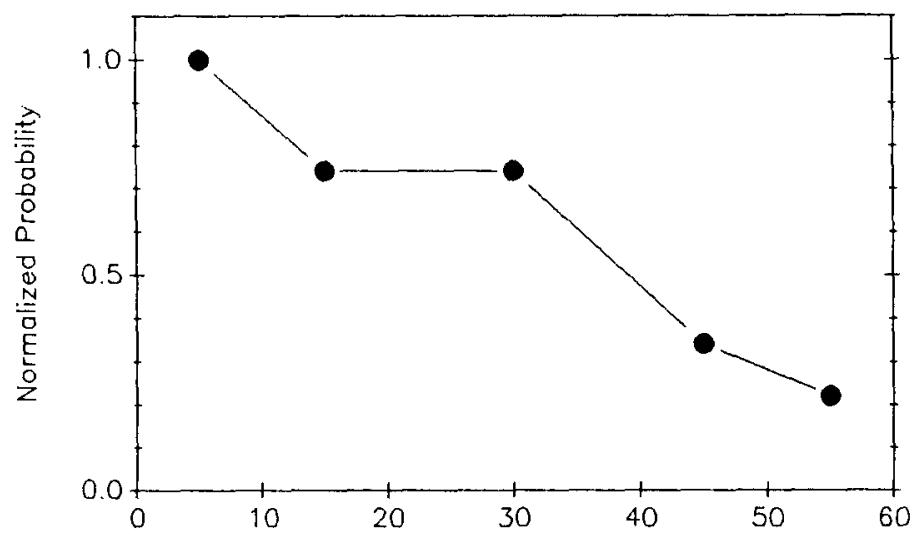

(a)

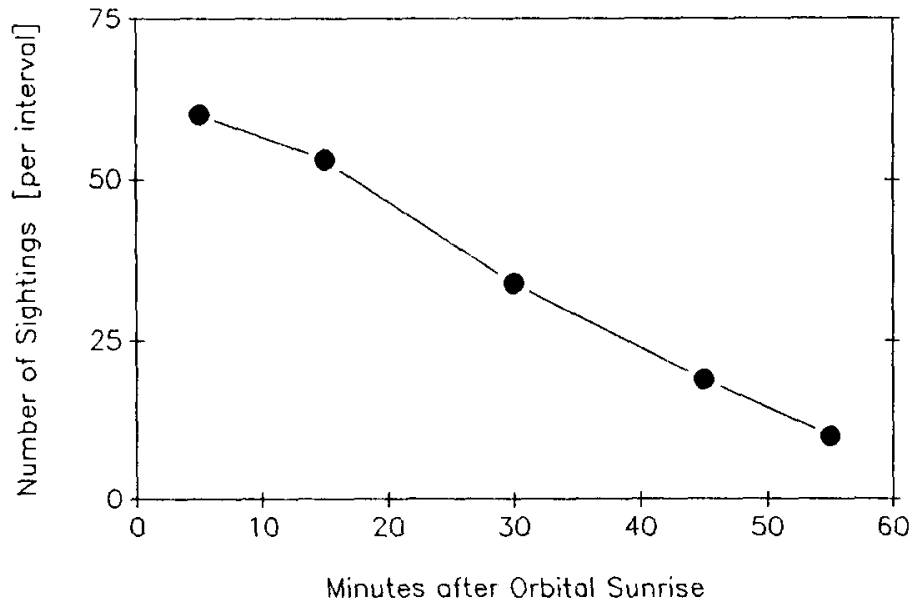

(b)

\section{FIGURE 2 - Sightings versus Orbital Phase}

(a) Normalized occurrence probability for the sightings versus time since orbital sunrise.

(b) As in 3(a) but absolute number of particle sightings summed for each time interval following orbital sunrise. 


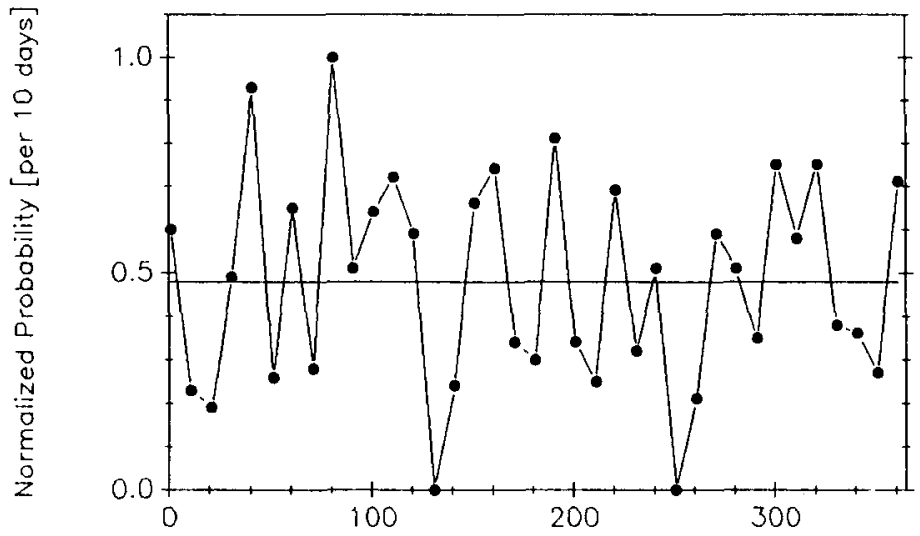

(a)

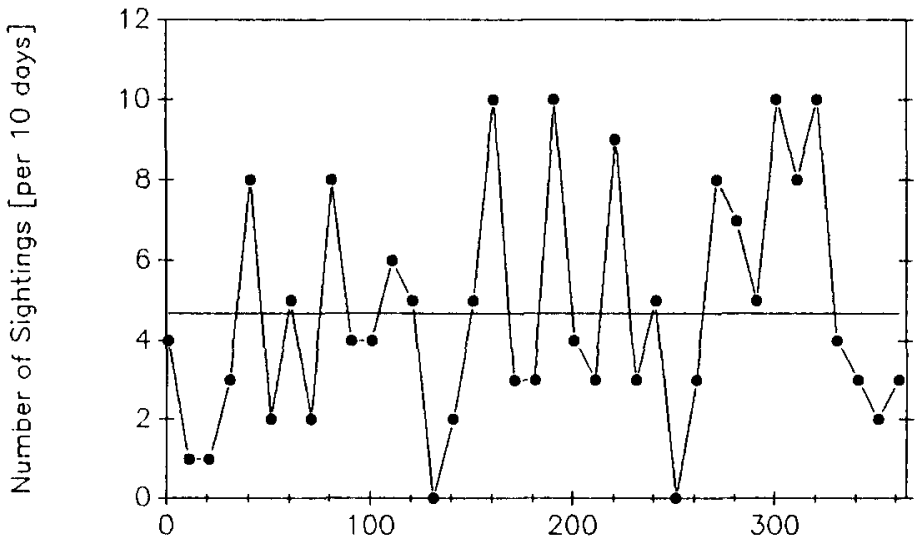

(b)

Doy of Year

IGURE 3 - Sightings versus Day of Year

(a) Normalized occurrence probability for the sightings versus day of the year (10 day intervals). The average normalized probability per 10 day interval $(0.48)$ is also shown.

(b) As in 4(a) but absolute number of particle sightings summed for each 10 day time interval (average $=4.7$ sightings per 10 day interval of DOY). 


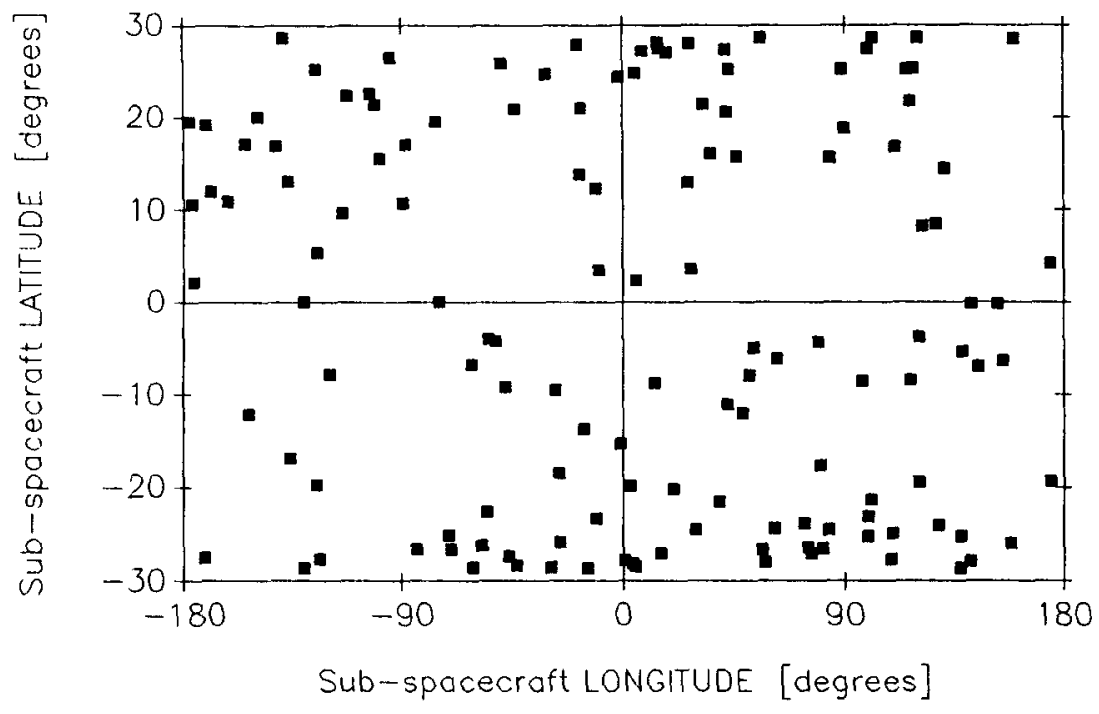

FIGURE 4 - Sub-spacecraft Latitude and Longitude. Each point here represents the sub-spacecraft latitude and longitude at the time of a contaminated image. 


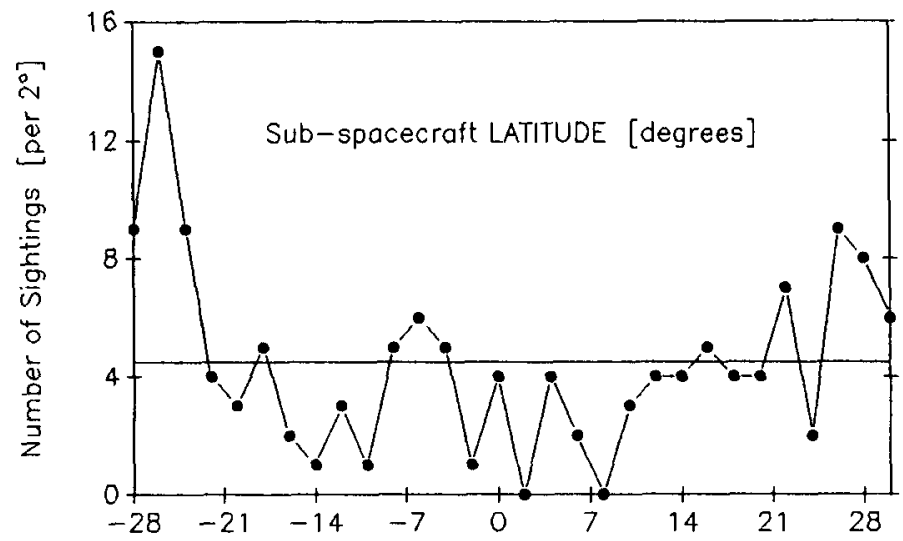

(a)

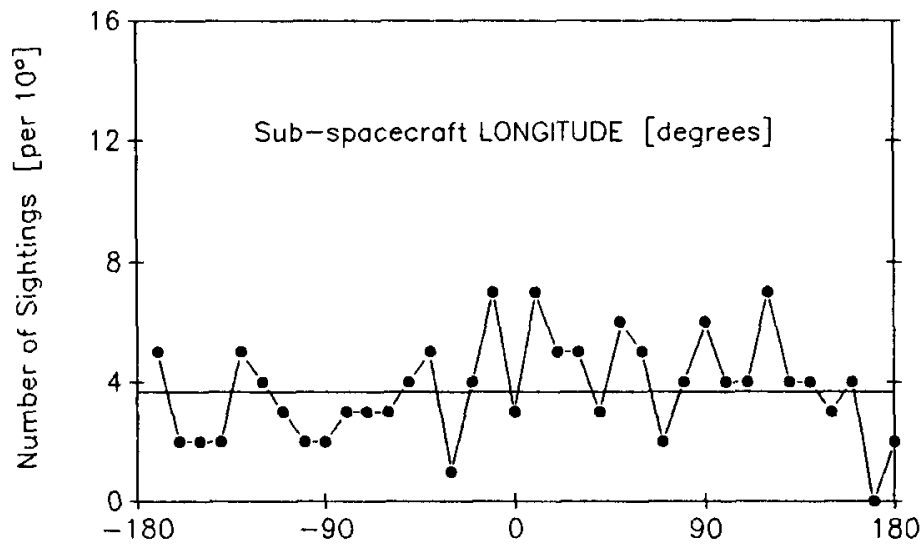

(b)

FIGURE 5 - Sub-spacecraft Latitude and Longitude.

(a) Absolute number of sightings per 2 degree interval of sub-spacecraft latitude (average $=4.5$ sightings per 2 degree interval of sub-spacecraft latitude).

(b) Absolute number of sightings per 10 degree interval of sub-spacecraft longitude (average $=3.7$ sightings per 10 degree interval of sub-spacecraft longitude). 